\title{
Luminous calibration of oscilloscopic displays
}

\author{
VINCENT DI LOLLO \\ University of Alberta, Edmonton, Alberta T6G 2E9, Canada
}

\begin{abstract}
Procedures are described for adjusting the intensity of computer-driven oscilloscopic displays. The adjustment maintains a fixed intensity level on different occasions and equates the brightness of brief stimuli displayed for different durations (i.e., compensates for the effects of time-intensity reciprocity).
\end{abstract}

Computer-controlled oscilloscopic displays have been employed with increasing frequency in studies of visual perception, but little attention has been paid to intensitycalibration and to brightness-equalization procedures. Most frequently, two separate adjustments are required: (1) A calibration procedure is needed to insure that the oscilloscope is set at a fixed level of intensity from day to day. (2) A brightness-equalization procedure may be needed to compensate for the effects of time-intensity reciprocity in instances where successive displays (or different portions of a given display) are plotted for different durations.

Described here are procedures adopted for meeting both types of calibration requirements (Di Lollo, 1977; Di Lollo, in press; Di Lollo \& Wilson, 1978). All stimuli are displayed on a Tektronix 602 oscilloscope, equipped with P15 phosphor under the control of a digital computer (PDP-8/L).

The calibration procedures do not provide absolute photometric measurements across a range of oscilloscopes or in different laboratories. Rather, the procedures are designed to enable maintenance of a given intensity setting from day to day and to permit brightness equalization of displays of different durations on a given display instrument. Reliability rather than validity is the principal object.

Three 8-bit digital-to-analog converters (X, Y, and $\mathrm{Z}$ ) plus additional steering logic were installed in the computer system to drive the display oscilloscope. The $\mathrm{X}$ - and $\mathrm{Y}$-axes define the Cartesian coordinates of each dot on the display surface and the Z-axis defines the intensity of each dot, from dimmest (invisible) to brightest (almost flaring), in 256 steps.

Modulation of the Z-axis is as follows: The output of the digital-to-analog converter attached to the $Z$-axis (Z-D/A) is in the form of a voltage level (a plateau) that can be set between 0 and $+5 \mathrm{~V}$, depending on the 8bit value that had been loaded from the accumulator. Even when the plateau is at maximum level, no dot is visible

This work was supported by the National Research Council of Canada, Grant A0269. Amedeo F. Sala Spini designed the display system. Requests for reprints should be sent to Vincent Di Lollo, Department of Psychology, Biological Sciences Building, University of Alberta, Edmonton, Alberta, Canada T6G 2E9. on the screen. Intensification is provided by a 1.6 -microsec pulse, fixed at $+5 \mathrm{~V}$, riding on the plateau level. Since the gain of the oscilloscope is set so that no visible display is produced by an input of $+5 \mathrm{~V}$ or less, an intensification pulse riding on a $0-\mathrm{V}$ plateau produces no visible result. But the dot becomes increasingly bright and ultimately reaches maximum intensity as the intensification pulse rides on a plateau whose level is increased to any of 256 values between 0 and $+5 \mathrm{~V}$.

Once set, the level of the plateau needs no resetting unless a new level of display intensity is required. When needed, a new level can be set simply by loading the new value into the computer's accumulator and transferring it to the Z-D/A by the appropriate input/output transfer instruction. Thus, the system provides the capability of changing the intensity of every individual dot in a given display, rapidly and under software control.

\section{INTENSITY CALIBRATION}

One method for calibrating the luminance of oscilloscopic displays uses the eye as a null meter to set a known reference source at the same luminance as that of a dot pattern plotted on the oscilloscope's surface (Sperling, 1971). An alternate method makes use of the Tektronix J16 digital photometer (Di Lollo, 1977; Di Lollo \& Wilson, 1978). The photometer is held within an aluminum and felt frame that hooks directly onto the face of the oscilloscope and excludes all extraneous light. The photometer's photosensitive probe has an acceptance angle of $8 \mathrm{deg}$ and is positioned, at a distance of $25 \mathrm{~mm}$, directly in front of a standard square test patch plotted on the display surface. The patch consists of 15 rows of 15 dots each, evenly spaced, which covers an area of $9.0 \times 9.0 \mathrm{~mm}$ in the center of the display surface. The display is continuously refreshed at the rate of $31.9 \mathrm{dots} / \mathrm{msec}$.

Calibration consists of adjusting the gain of the oscilloscope so that the standard patch, plotted at the same $\mathrm{Z}$ value on every occasion, yields a reading of $.024 \mathrm{~lm} / \mathrm{m}^{2}$ on the lux scale of the photometer. The lux scale is used in preference to the more appropriate nits scale $\left(\mathrm{cd} / \mathrm{m}^{2}\right)$ because the illuminance probe permits greater resolution. The value of .024 lux provides a comfortable level of visibility. 


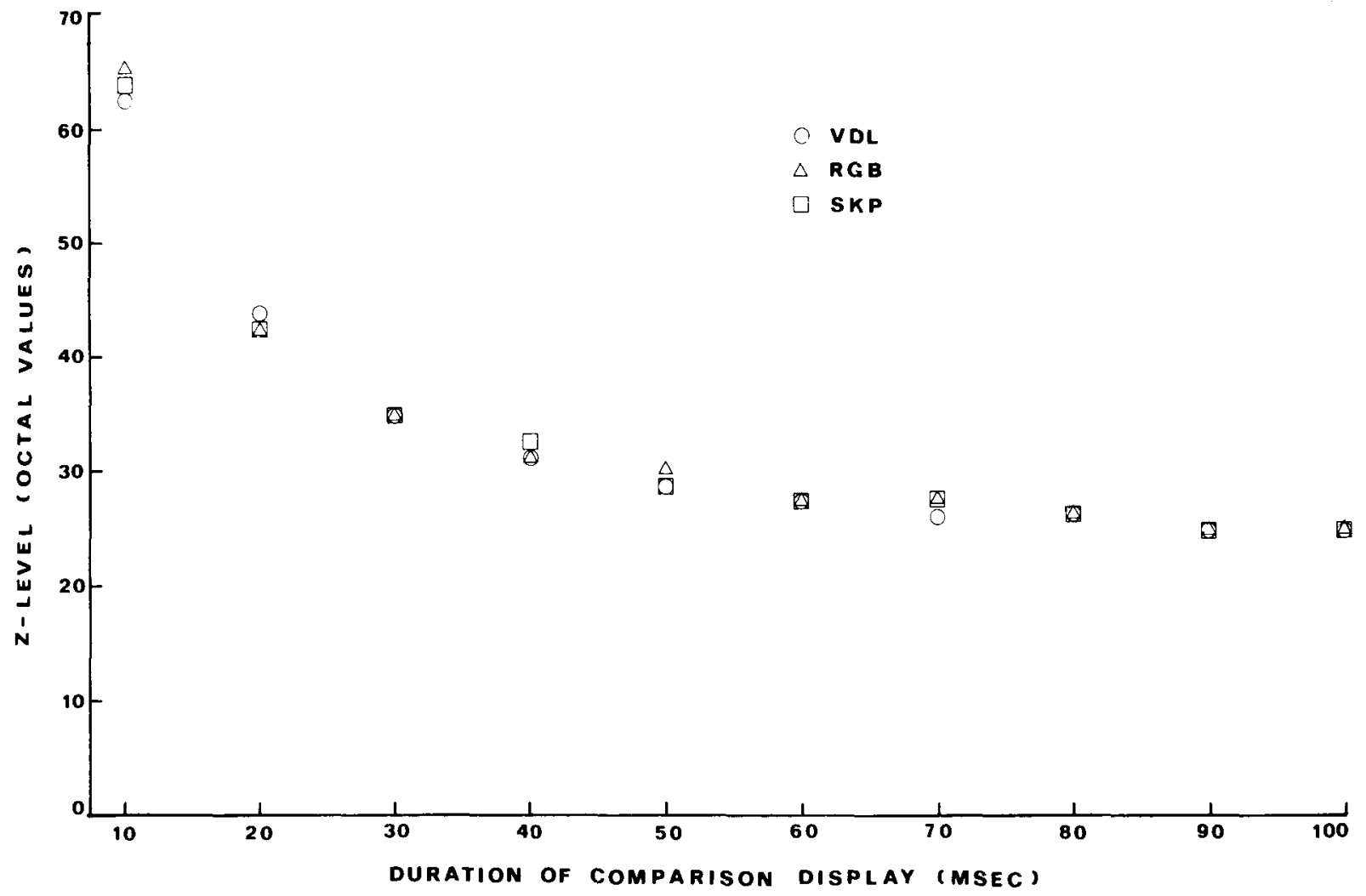

Figure 1. Intensity levels (in arbitrary $Z$ units) to which each subject set a comparison stimulus, plotted for the duration shown on the abscissa, so that it appeared of the same brightness as a standard stimulus plotted for 100 msec at an arbitrary $Z$ value of 24 . Each point on the curve defines a combination of intensity and duration that yields the same brightness as does any other point on the curve.

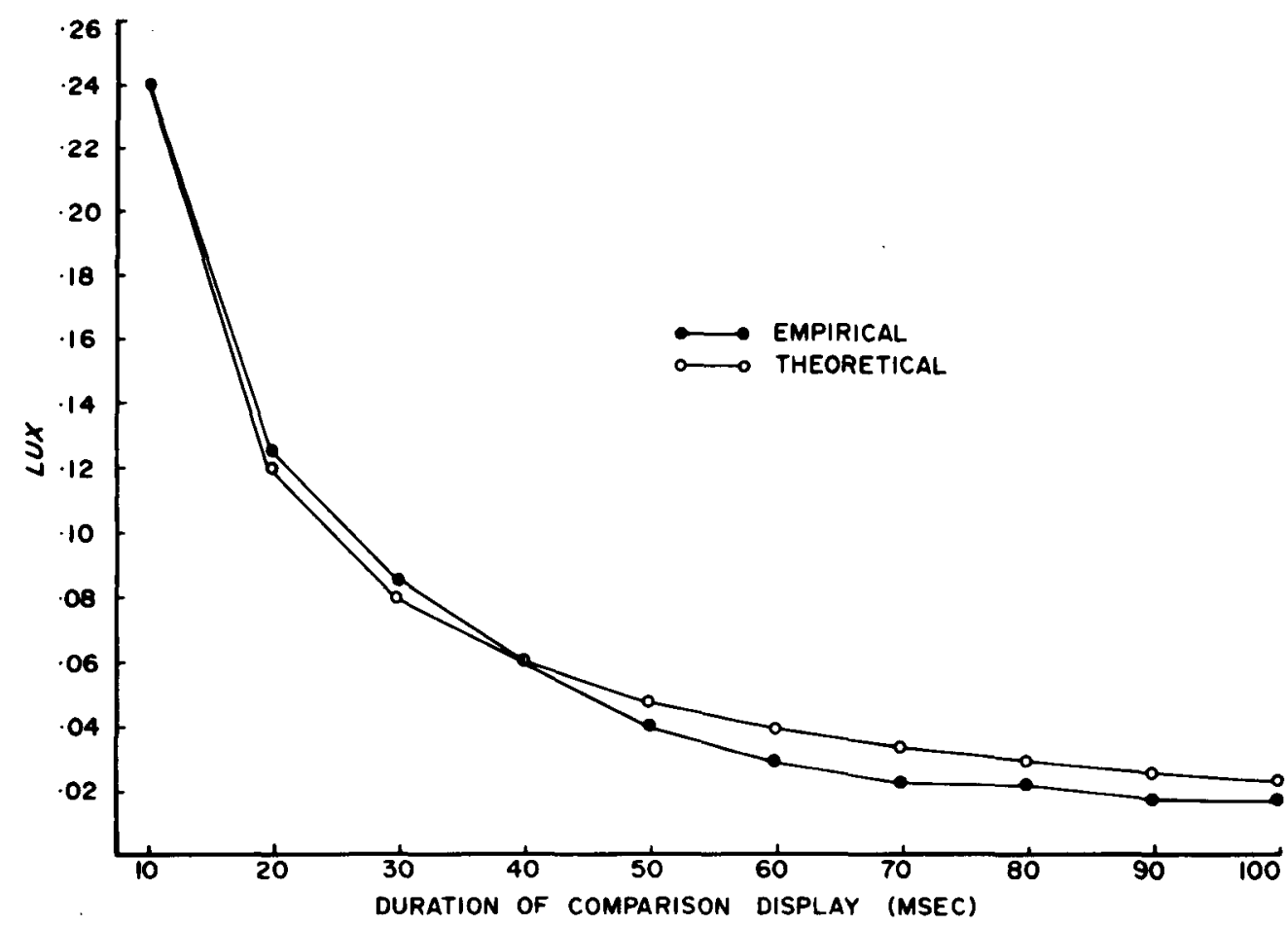

Figure 2. Comparison between empirically based equal-brightness values derived from the data reported in Figure 1 and equivalent theoretical values computed on the basis of a multiplicative relationship between intensity and duration. 
This calibration procedure requires a few minutes and can be carried out easily. Since the purpose is simply to maintain a fixed intensity setting, choice of a specific plotting rate for displaying the test patch is not critical although it should be reported. Similarly, the value and the scale (lux or nits) to which the instrument is calibrated is at the discretion of the user. Plotting rate, as well as focusing and astigmatism of the electron beam, should not vary between calibrations.

\section{BRIGHTNESS EQUALIZATION}

In the experiments cited above, most stimuli consisted of two separate configurations displayed successively for durations between 10 and $200 \mathrm{msec}$. In some conditions the two portions of a given stimulus were displayed for different durations. This made the longer stimulus appear brighter, as would be expected on the basis of time-intensity reciprocity for stimulus durations up to about $100 \mathrm{msec}$ (Bloch's law; see Butler, 1975). The brightness mismatch was experimentally undesirable and was averted by plotting the briefer displays at higher levels of intensity (i.e., at higher $Z$ levels).

Procedures for estimating the appropriate $\mathrm{Z}$ levels were almost identical to the procedure independently developed by Serviere, Miceli, and Galifret (1977) for a similar purpose. The plan was to establish a $Z$ level at which a display was comfortably visible when plotted for $100 \mathrm{msec}$ and then to find $Z$ levels at which briefer displays would appear of the same brightness as the 100-msec display.

Two displays consisting of 12 dots each (arranged in three columns of four dots each) were shown side by side in the center of the display area. The dots were evenly spaced so that the combined displays subtended an angle of approximately 1.5 deg vertically and $2.4 \mathrm{deg}$ horizontally. The display was plotted at a rate of $12.4 \mathrm{dots} / \mathrm{msec}$.

Duration of the standard (left-hand) display was $100 \mathrm{msec}$; intensity was fixed at a $\mathrm{Z}$ value which yielded a comfortably visible display. The comparison (righthand) display was shown for the duration for which the appropriate $Z$ value was being sought. The initial $Z$ value of the comparison display was set at random between 0 and 256. The subject's task was to push a button to generate both displays simultaneously and then to push one of two other buttons to increase or decrease the $\mathrm{Z}$ value of the comparison display. Unwanted apparentmotion effects were avoided by plotting the comparison display halfway through the duration of the standard display. The subject adjusted the luminance of the comparison display until both displays appeared of equal brightness. Depression of a fourth button terminated the trial. Every subject made five settings for each of the 10 comparison-stimulus durations between 10 and $100 \mathrm{msec}$ in rand omly sequenced steps of $10 \mathrm{msec}$. The $Z$ settings thus obtained were highly reliable be tween subjects, as well as for the same subject on separate occasions. In Figure 1 the results of the matching procedure are shown separately for each of three subjects. On the basis of time-intensity reciprocity, briefer displays required higher $Z$ values.

There was close correspondence between luminance values obtained theoretically (i.e., on the basis of a simple multiplicative relationship between intensity and duration) and empirically. Figure 2 compares computed theoretical values with empirical values derived from the data reported in Figure 1. The empirical values in Figure 2 were obtained by employing the lux scale $\left(\mathrm{lm} / \mathrm{m}^{2}\right)$ of the digital photometer to measure the intensity of the standard square test patch plotted continuously on the display surface. Each empirical point in Figure 2 represents a separate photometric reading of the test patch plotted at the average $Z$ value that had been obtained in the preliminary study for the duration of comparison stimulus stated on the abscissa.

Each of the subjects in the experiments cited earlier was required to participate in a preliminary study where the above matching procedures were carried out. In the experiment proper, all displays lasting up to $100 \mathrm{msec}$ were plotted at the average $Z$ value that had been obtained for the appropriate comparison-stimulus duration in the preliminary study.

A requirement of this procedure is that the plotting rate of the stimuli displayed in the experiment proper be the same as that utilized in establishing the equal. brightness contour in the preliminary study. In other respects, the procedure seems to be exceptionally sturdy. The visual configuration used in the brightnessequalization procedure can be varied to match that used in the experiment proper. No requirement for linearity is made with respect to any part of the system. Indeed, the function relating brightness of display to $Z$ setting will depend on a variety of factors, such as linearity of the Z-D/A and the characteristics of the phosphor on the display surface. But the shape of the function is irrelevant, because the $Z$ value for every stimulus duration is defined empirically and independently from all other $\mathrm{Z}$ values. In this sense, even a nonmonotonic function, unlikely though it may be, would not invalidate the procedure.

\section{REFERENCES}

Butler, T. W. Luminance-duration relationships in the photopic ERG and the apparent brightness of flashes. Vision Research, $1975,15,693-698$.

Di Lollo, V. Temporal characteristics of iconic memory. Nature, $1977,267,241-243$.

Di LoLLo, V. Temporal integration in visual memory. Journal of Experimental Psychology: General, in press.

Di LolLo, V., \& Wilson, A. E. Iconic persistence and perceptual moment as determinants of temporal integration in vision. Vision Research, 1978, 18, 1607-1610.

Serviere, J., Miceli, D., \& Galifret, Y. A psychophysical study of the visual perception of "instantaneous" and "durable." Vision Research, 1977, 17, 57-63.

SPE Rling, G. The description and luminous calibration of cathode ray oscilloscope visual displays. Behavior Research Methods \& Instrumentation, 1971, 3, 148-151.

(Received for publication February 8, 1978; revision accepted May $17,1979$. 